\title{
Internet na promoção da saúde: um instrumento para o desenvolvimento de habilidades pessoais e sociais
}

I ${ }^{1}$ Helena Beatriz da Rocha Garbin, ${ }^{2}$ Maria Cristina Rodrigues Guilam,

\author{
${ }^{3}$ André Faria Pereira Neto I
}

Resumo: O desenvolvimento de habilidades pessoais e sociais, no sentido da aquisição de maior controle e maior poder de decisão (Empowerment), é considerado estratégia de fundamental importância na promoção da saúde. $\mathrm{O}$ acesso à informação é uma das bases para tal. Muito vem sendo dito e escrito sobre o imenso potencial da internet, estudos apontam-na como um instrumento especial para obtenção de informação e de capacitação, tanto individual, quanto comunitária. Este texto se apoia na literatura científica nacional e internacional sobre promoção da saúde, informação e saúde na internet e divisão/exclusão digital, com o objetivo de discutir a possibilidade de utilização da rede como recurso em projetos voltados para a promoção da saúde, apontando pontos positivos e possíveis barreiras, com destaque para a questão da divisão/exclusão digital de boa parte da população brasileira. Os autores consideram que a internet pode vir a ser uma grande aliada na construção de projetos de promoção da saúde. No entanto, no desenvolvimento desses projetos, é fundamental considerar os potenciais riscos e compreender que as estratégias e programas na área da promoção da saúde precisam se adaptar às necessidades e possibilidades de cada local e levar em conta as diferenças sociais, culturais e econômicas.

\author{
1 Doutoranda pela Escola \\ Nacional de Saúde Pública/ \\ FIOCRUZ. Endereço eletrônico: \\ helena.garbin@ensp.fiocruz.br \\ 2 Pesquisadora (ENSP/ \\ FIOCRUZ). Doutora em Saúde \\ Coletiva (IMS/UERJ). Endereço \\ eletrônico: guilam@ensp. \\ fiocruz.br \\ ${ }^{3}$ Pesquisador (ENSP/FIOCRUZ). \\ Doutor em Saúde Coletiva (IMS/ \\ UERJ). Endereço eletrônico: \\ apereira@fiocruz.br
}


O desenvolvimento de habilidades pessoais e sociais é considerado estratégia de fundamental importância na promoção da saúde. Ele pode ser alcançado "através da divulgação de informação, educação para a saúde e intensificação das habilidades vitais" (BRASIL, 2002, p.14). O conceito de empoderamento (empowerment), tanto individual, quanto coletivo, perpassa essa estratégia de forma explícita (BUSS, 2003; CARVALHO, 2004). Ele reitera a importância do incremento das possibilidades de controle dos indivíduos e das comunidades sobre sua própria saúde e sobre o meio ambiente, conforme defende a Carta de Ottawa (1986). O documento afirma que: "As pessoas não podem realizar completamente seu potencial de saúde se não forem capazes de controlar os fatores determinantes de sua saúde..." (BRASIL, 2002, p.21). Nos diversos documentos advindos das conferências internacionais que se seguiram à de Ottawa, observase a reafirmação da importância do acesso à informação para a capacitação do indivíduo e da comunidade, no sentido da aquisição de maior controle e maior poder de decisão. A "Declaração de Jacarta”, realizada em 1997, pode servir de exemplo neste sentido: "[...] aprender sobre saúde fomenta a participação. $\mathrm{O}$ acesso à instrução e à informação é essencial para alcançar a participação eficaz e o direito de voz das pessoas e das comunidades" (BRASIL, 2002, p.50).

Diversos são os meios para acesso e obtenção de informações sobre saúde, e, nas últimas três décadas, em função de intensas transformações sociais, políticas, econômicas, tecnológicas, culturais, entre outras, passamos a viver num mundo globalizado, altamente tecnológico, no qual a informação tornou-se capital fundamental e o acesso a ela é cada vez mais rápido e fácil (GIDDENS, 2002). Dentre as diversas mídias, como a televisão, o rádio e o jornal, a internet se destaca em função da infinidade de possibilidades, do estímulo à postura ativa do usuário e da oportunidade que ele tem de produzir a informação, em vez de simplesmente acessá-la. O uso da internet como fonte de informaçôes vem crescendo quase exponencialmente desde que ela veio a público em 1991 e, em especial, desde que foram lançados os primeiros navegadores (browsers) em 1993 (HART, 2004). Há pouco mais de 10 anos, Castells (1996) defendia que o mundo estava entrando na "Era da Informação" e que a internet produziria um enorme impacto na sociedade, tanto no nível individual, quanto nas formas de organização social. Avaliação semelhante foi feita por Hart (2004): 
Quando a internet veio a público, em 1993, havia somente 3 milhões de usuários

no mundo todo. Nos 5 anos seguintes, o número de usuários aumentou para 100 milhōes. Esse número é atualmente estimado em 600 milhōes, ou uma em cada dez pessoas vivas. Nenhuma outra tecnologia anterior se difundiu tão rapidamente através da população mundial (HART, 2004: 2).

O objetivo deste artigo é discutir a possibilidade de utilização da internet como recurso em projetos voltados para a promoção da saúde, apontando pontos positivos e possíveis barreiras, com destaque, no caso brasileiro, para a questão da divisão digital.

\section{Considerações metodológicas}

Este estudo se apoia na literatura científica nacional e internacional sobre promoção da saúde, informação sobre saúde na internet e divisão/exclusão digital. O trabalho adota como referencial a "Nova Promoção da Saúde", os documentos oficiais (cartas e declaraçôes) construídos nas Conferências Internacionais da Promoção da Saúde, a partir da Conferência Internacional sobre Promoção da Saúde de Ottawa (1986). Utiliza-se, ainda, a literatura internacional sobre informação e saúde na internet para análise de pontos positivos e negativos de seu uso. A análise da situação de inclusão/exclusão digital no Brasil foi feita com base na 5a Pesquisa sobre o Uso das Tecnologias da Informação e da Comunicação no Brasil - TIC DOMICÍLIOS, conduzida pelo Núcleo de Informação e Coordenação do Ponto BR (NIC.br), braço executivo do Comitê Gestor da Internet no Brasil (CGI.br).

\section{Promoção da Saúde}

A compreensão do processo saúde-doença como um processo dinâmico, que sofre influência de fatores sociais, econômicos, culturais, laborativos, ambientais, étnicos ou raciais, psicológicos e comportamentais (determinantes sociais/ condições de vida) teve sua origem nos séculos XVIII e XIX, particularmente nas obras de Virchow, Chadwick, Villermé (ROSEN, 1983; CZERESNIA, 2003/2009; MARCONDES, 2004; BUSS, 2007). A concepção da determinação social perde força a partir (principalmente) do surgimento da bacteriologia, quando a medicina adquire o monopólio científico e legal, e o poder médico se estabelece e se impõe, tanto na assistência como nas questôes relacionadas à saúde pública (ROSEN, 1983; QUEIRÓZ, 1986). Após a 2a Guerra Mundial, 
entretanto, essa concepção retomou força e passou a ser incrementada em vários países (CZERESNIA, 2003/2009; BUSS, 2007). As práticas de saúde correspondentes a essa compreensão têm por objetivo não apenas intervir na doença, mas, sobretudo, atuar na melhoria das condiçôes concretas e reais de vida de um indivíduo ou de uma coletividade. Nessa perspectiva, os problemas de saúde deixam de ser exclusivamente de competência médica e admitem estratégias de intervenção para os quais concorrem outros segmentos da sociedade.

Documentos precursores evidenciam as relações entre saúde e qualidade/ condições de vida, como o Relatório Lalonde, de 1974 (Canadá), a Conferência Internacional de Atenção Primária de Saúde de Alma-Ata, de 1977, o Relatório Black sobre as Desigualdades em Saúde, de 1980 (Inglaterra), a VIII ${ }^{a}$ Conferência Nacional de Saúde, de 1986 (Brasil), entre outros (BUSS, 2000; MARCONDES, 2004). No entanto, a Carta de Ottawa, de 1986 (BRASIL, 2002), e o Relatório Final da I ${ }^{\text {a }}$ Conferência Internacional sobre Promoção da Saúde, realizada nessa cidade, são considerados como documentos fundadores da "Nova Promoção da Saúde”. Esse novo conceito tem uma breve história, que merece ser resgatada.

A primeira Conferência Internacional sobre Promoção da Saúde, realizada em 1986 na cidade de Ottawa (Canadá), promulgou a "Carta de Ottawa", segundo a qual:

Promoção da saúde é o nome dado ao processo de capacitação da comunidade para atuar na melhoria de sua qualidade de vida e saúde, incluindo uma maior participação no controle deste processo [...]. [A] saúde deve ser vista como um recurso para a vida, e não como objetivo de viver (BRASIL, 2002, p.19).

A carta define novas condições e recursos fundamentais para a saúde, como a garantia da paz, da habitação, da educação, da alimentação, da renda, do ecossistema estável, dos recursos sustentáveis, da justiça social e da equidade. Para que sejam atingidas tais metas, foram pensadas três principais estratégias, a saber: defesa da saúde, capacitação e mediação (BRASIL, 2002, p.20).

A defesa da saúde parte do princípio de que ela "é o maior recurso para o desenvolvimento social, econômico e pessoal, assim como uma importante dimensão da qualidade de vida” (BRASIL, 2002, p.20). O documento considera primordial que se caminhe no sentido de alcançar a equidade, para que todos tenham a possibilidade de desenvolver ao máximo sua saúde potencial. Por meio de uma aproximação positiva da saúde, considera-se o processo de saúde-doença como um processo dinâmico atrelado aos determinantes sociais e, portanto, 
às condições de vida de uma dada população, num dado momento histórico.

Nesse sentido, são considerados fundamentais "os ambientes favoráveis, o acesso à informação, as experiências e habilidades na vida, [...] oportunidades que permitam fazer escolhas por uma vida mais sadia” (BRASIL, 2002, p.21) e a mediação de diversos setores da sociedade, além do setor saúde.

Foram propostos cinco campos centrais de ação, a saber: construção de políticas públicas saudáveis; criação de ambientes favoráveis à saúde; desenvolvimento de habilidades individuais e sociais; reforço da ação comunitária; e reorientação dos serviços de saúde (BRASIL, 2002). A organização do trabalho em saúde, nesse modelo, passou a incorporar novas tecnologias como a comunicação, e baseou-se cada vez mais na interdisciplinaridade, nas ações intersetoriais, na educação e na criação de mecanismos de controle social coletivo, por intermédio de ações com a participação da sociedade civil. Sobre o tema, Buss afirma:

A promoção da saúde, como vem sendo entendida nos últimos 20-25 anos, representa
uma estratégia promissora para enfrentar os múltiplos problemas de saúde que afetam
as populações humanas e seu entorno neste final de século. Partindo de uma concep-
ção ampla do processo saúde-doença e de seus determinantes, propõe a articulação
de saberes técnicos e populares, e a mobilização de recursos institucionais e comuni-
tários, públicos e privados, para seu enfrentamento e resolução (BUSS, 2000, p.165)

A partir da leitura dos documentos construídos nessas conferências internacionais, assim como dos demais textos de referência sobre o tema, fica claro que essa é uma tarefa que pode (e deve) ser realizada nos locais de trabalho, nas residências, nas escolas, em associações comunitárias, e também em unidades de saúde. Ou seja, a implementação dessas estratégias, bem como de todas as estratégias da promoção da saúde, não é exclusividade desse setor.

No nosso entender, as estratégias e campos de ação propostos pela "Carta de Ottawa", ratificados em outros eventos, estão profundamente entrelaçados. No entanto, neste artigo daremos destaque a um deles: o desenvolvimento de habilidades pessoais e sociais.

\section{A internet como recurso}

Muito vem sendo dito e escrito sobre o imenso potencial da internet de possibilitar o empowerment dos indivíduos que a utilizam. Estudos apontam-na como um instrumento especial para obtenção de informação/conhecimentos e de capacitação, tanto individual, quanto comunitária (HARDEY, 1999; HARDEY, 
2001; EYSENBACH et al., 2001; BENIGERI et al., 2003; GINMAN et al., 2003; KORP, 2004; ZIEBLAND, 2004a; GRIERSON et al., 2006).

De fato, a internet é a mais completa e complexa fonte de informações na atualidade. Com seus milhares de sites relacionados à saúde, permite aos indivíduos o acesso a todo tipo de informação, desde a busca diagnóstica até compra de medicamentos, passando por orientação terapêutica, sem esquecer as páginas pessoais e as comunidades virtuais (NETTLETON et al., 2005). Além disso, é possível obter essas informações a qualquer hora, em qualquer lugar, fácil e rapidamente.

Estudos realizados em diversos países, incluindo o Brasil, mostram o crescente uso da internet para a obtenção de informação sobre saúde. Apesar de alguns estudos apontarem que homens têm mais acesso à internet do que mulheres (SORJ et al., 2005; WILSON, 2007), no caso específico da busca de informaçôes sobre saúde, o grupo que se destaca é o de mulheres com menos de 55 anos e com alto nível educacional (BACKA, 2003; GINMAN, 2003; WILSON, 2007). Mais recentemente, vem apresentando significativo crescimento o grupo dos indivíduos da terceira idade (ALPAY, 2004; WILSON, 2007).

$\mathrm{O}$ fato de adquirir expertise sobre sua condição e ter participação ativa em seu tratamento (PANDEY et al., 2003; ZIEBLAND et al., 2004b), reafirma a condição do indivíduo de membro competente da sociedade. O recurso à internet é tido pelo paciente como forma de estar preparado para fazer perguntas, compreender o que é dito, e, consequentemente, sentir-se mais confiante, com maior controle sobre sua saúde, sobre seu tratamento e, enfim, sobre sua vida (NETTLETON et al., 2005). Giddens (2002) defende que a população vem vivendo um processo de requalificação pelo amplo acesso à informação.

É mais provável que um indivíduo acometido por uma patologia busque informações sobre saúde na rede do que um indivíduo sadio (NETTLETON, 2004; BERGER et al., 2005; BUNDORF et al., 2006). Portadores de patologias consideradas estigmatizantes (depressão, incontinência urinária, herpes, entre outras) encontram no anonimato das relaçóes virtuais um caminho, pois a possibilidade de falar sobre suas queixas aumenta consideravelmente com a comunicação à distância, tanto com médicos quanto com outros indivíduos portadores de quadros semelhantes. O risco de discriminação, embaraço e humilhação é drasticamente reduzido (BERGER et al., 2005). Ao mesmo tempo, 
pode-se fazer uso da internet para a aquisição de informações sobre questôes

relativas à saúde com o objetivo de seguir um estilo de vida saudável, revelando uma postura pró-ativa, de autocuidado em relação à saúde (PANDEY et al., 2003).

Comunidades virtuais são fontes não só de informação e conhecimento (técnico ou leigo), mas também possibilitam a formação de redes sociais de suporte (ZIEBLAND et al., 2004b; FOX et al., 2005a; FOX et al., 2005b). Mais ainda, grupos organizados em comunidades virtuais (ou em associaçôes de doentes crônicos), como os de indivíduos ostomizados, ou acometidos por diabetes, HIV/AIDS, Esclerose Múltipla, entre muitas outras, podem ser fontes de poder e de pressão sobre o campo da saúde, aí incluído o Estado, as corporações e as empresas privadas (GILLET, 2003).

Diversos são também os pontos negativos do uso da internet, a começar pela qualidade da informação que nela é oferecida, muitas vezes incompleta, contraditória, incorreta, excessiva ou atéfraudulenta. O "Dilúvio de Informações" (LEVY, 1993) a que os indivíduos são expostos pode gerar confusão, ansiedade e ainda mais incerteza. Pode gerar, enfim, redução da confiança e do controle. A dificuldade de compreender as informações encontradas, ou ainda separar a boa informação da informação ruim, é uma questão importante, em especial em países onde a população apresenta baixos níveis de escolaridade (CASTIEL et al., 2003; ZIEBLAND, 2004a; NETTLETON et al., 2005; SEALE, 2005). Alguns autores destacam o potencial medicalizante da internet (GINMAN, 2003; KORP, 2006; BROOM et al., 2008), na medida em que se entende medicalização como a compreensão crescente de diversos aspectos da vida diária, comportamentos e atitudes em termos médicos (ZOLA, 1972; CONRAD, 1992; CONRAD, 2007).

No entanto, a principal barreira para a plena utilização da internet em projetos de promoção da saúde, em especial com foco no desenvolvimento de habilidades pessoais por meio da informação e educação em saúde, ainda está na divisão/exclusão digital de boa parte da população brasileira (CASTIEL et al., 2003; SORJ et al., 2005).

\section{Internet no Brasil}

O Brasil tem apresentado um sensível crescimento no número de residências com acesso à internet nos últimos anos. Foi realizada em 2009, em todo o 
território nacional, a 5a Pesquisa sobre o Uso das Tecnologias da Informação e da Comunicação no Brasil - TIC DOMICÍLIOS, pelo Núcleo de Informação e Coordenação do Ponto BR (NIC.br), braço executivo do Comitê Gestor da internet no Brasil (CGI.br). Aproximadamente 36\% dos domicílios possuem computador de mesa, contra 24\% em 2007 e 28\% em 2008 (Tabela 1). O uso do computador está relacionado principalmente ao grau de instrução, à faixa etária e à renda familiar do domicílio.

O crescimento mais expressivo se deu em domicílios cuja renda está entre 3 e 5 salários mínimos, nos quais a penetração passou de $23 \%$ para $40 \%$ no período. Essas famílias são justamente o alvo dos programas de incentivo fiscal do Governo Federal (BRASIL, 2008).

Estão conectados à internet 27\% dos domicílios (em torno de 13 milhôes), contra 14\% em 2006 e $20 \%$ em 2008 (Tabela 1). A pesquisa revela as desigualdades regionais no país. Nas regiōes Sul e Sudeste, possuem computadores conectados à internet mais de 30\% dos domicílios (acima da média nacional), enquanto nas regiōes Norte e Nordeste, somente 13\% dos domicílios possuem computador. Em torno de $45 \%$ dos brasileiros já fizeram uso da internet pelo menos uma vez. Os principais motivos alegados para a falta de acesso nos domicílios que possuem computador são o alto custo (48\%), a possibilidade de acesso em outro local (22\%), a falta de necessidade ou interesse $(13 \%)$ e a falta de habilidade para usar a internet $(8 \%)$.

Tabela 1. Domicílios com computador e acesso à internet no Brasil

\begin{tabular}{|c|c|c|}
\hline \multicolumn{3}{|c|}{ PERCENTUAL SOBRE O TOTAL DE DOMICÍLIOS } \\
\hline ANO & COMPUTADOR & INTERNET \\
\hline 2005 & $17 \%$ & $13 \%$ \\
\hline 2006 & $20 \%$ & $14 \%$ \\
\hline 2007 & $24 \%$ & $17 \%$ \\
\hline 2008 & $28 \%$ & $20 \%$ \\
\hline 2009 & $36 \%$ & $27 \%$ \\
\hline
\end{tabular}

Segundo a $5^{\text {a }}$ Pesquisa sobre o Uso das Tecnologias da Informação e da Comunicação no Brasil (BRASIL, 2010), o percentual dos usuários que 
acessam a internet de suas residências cresceu de $42 \%$ para $48 \%$ em relação a

2008, assim como o acesso na residência de outra pessoa (de 22\% para 26\%). Porém, o percentual dos que o fazem de centro público pago (lan house) teve queda de $48 \%$ para $45 \%$.

Existe uma clara relação inversa entre nível de renda e escolaridade quanto ao uso da internet em lan houses. As classes D e E são os principais usuários dos centros públicos, gratuitos ou pagos: "São numerosos os exemplos de favelas que incorporaram a cultura da internet em suas comunidades, justamente através das pequenas lan houses" (BRASIL, 2008; p.48). Foi observado um crescimento significativo do percentual de usuários que usam centros públicos gratuitos, entre 2005 e 2007, de 2\% para 6\%. Nos últimos dois anos, esse índice encontrava-se estável em $4 \%$.

Silva (2006) afirma que o país vem investindo em infraestrutura digital e já é o segundo na América Latina em competitividade de infraestrutura de rede, e o que mais cresce em investimentos para implantação de redes de computadores (130\% ao ano): "Somos o oitavo país em serviços governamentais online e o décimo país em serviços de rede informatizada e internet para pesquisas e em uso de redes governamentais” (SILVA, 2006, p. 116). Bechara (2008), que é consultor jurídico do Ministério das Comunicações e conselheiro do CGI.br, acredita que:

as dimensões e os desafios do Brasil não comportam soluções únicas e centralizadas para a erradicação da exclusão digital. Contudo, as experiências bem sucedidas demonstram que ações coordenadas dos diversos agentes públicos e privados são indubitavelmente o melhor caminho. É assim que ampliamos os espaços da inclusão (BECHARA, 2008; p.50).

Exemplos disso podem ser encontrados no trabalho de organizações não governamentais (ONG), como o Comitê pela Democratização da internet (CDI), com Escolas de Informática e Cidadania (EIC) espalhadas por comunidades de baixa renda no Brasil e em outros 10 países, e também pelo Viva Rio, com suas Estações Futuro, oferecendo acesso a cursos de informática e ao uso da internet a baixo custo.

As principais atividades desenvolvidas na internet, observadas no TIC Domicílios, são a comunicação (90\%), o lazer (86\%), a busca de informações e de serviços online (89\%) e a educação (72\%) (BRASIL, 2010). Do total de usuários da internet, 39\% apontaram a busca de informaçôes relacionadas à saúde e de serviços de saúde como atividade desenvolvida online, um crescimento 
de $6 \%$ em relação a 2008. É possível que a pandemia de gripe H1N1 tenha papel importante nesse salto. Observa-se nesse grupo um percentual acima da média nacional para as regiōes Centro-Oeste, Sudeste e Norte, bem como uma preponderância do sexo feminino sobre o masculino, e dos indivíduos trabalhando sobre os desempregados ou que não pertencem à população economicamente ativa. Quanto maior a faixa etária, o nível educacional, a classe social e a faixa de renda, maior o uso da internet para obtenção de informações sobre saúde.

\section{Inclusão/exclusão digital}

É fundamental entender que diferenças culturais e societárias, além das econômicas, modelam esse processo, que se desenrola em cada lugar, em cada grupo social, em cada região de cada país do globo de acordo com sua inserção. Diferentes estruturas sociais, políticas e econômicas determinam diferentes formas e possibilidades de obtenção de informação. Diferenças essas que também se refletem na informação que é ofertada na rede. Nem tudo está disponível na internet, e o que está disponível está sujeito a interesses hegemônicos, como, por exemplo, o favorecimento da língua inglesa e culturas correspondentes (CUNHA, 2008). Os caminhos mais utilizados para obter informações na internet, buscadores como Google, Yahoo, entre outros, tendem a oferecer, nos primeiros lugares, páginas de grandes organizações (SEALE, 2005).

No Brasil, apesar do crescimento apontado por pesquisas recentes, mais do que uma divisão digital, temos exclusão de boa parte da população. Estudo da Fundação Getúlio Vargas (apoiado na Pnad - IBGE, 2004) sugere que os fatores determinantes da exclusão digital são semelhantes aos fatores determinantes da exclusão social.

Tal observação não é exclusiva da sociedade brasileira. Estudos realizados nos primeiros anos do século XXI nos Estados Unidos da América mostraram uma visão semelhante sobre a relação entre a exclusão digital e a exclusão social (GIBBONS, 2000; COTTEN et al., 2004). Em nosso país, o acesso a internet ainda é limitado a uma minoria. Os altos custos do computador e do acesso à rede, aliados à residual escolaridade da ampla maioria da população são barreiras significativas.

Contudo, é complexa a análise de exclusão/inclusão digital (SORJ et al., 2005). Pode-se destacar, primeiramente, a diferença entre a telefonia e a internet. Apesar 
de pertencerem ao mesmo grupo de Tecnologias de Informação e Comunicação (TIC), a internet é excludente para analfabetos, o que não ocorre com o telefone comum (mas que talvez venha a ocorrer com o celular) (SORJ et al., 2005). Uma segunda questão, bastante polêmica, diz respeito ao tão badalado potencial democratizante e equalizante da internet. Sorj \& Guedes (2005) não são entusiastas dessa visão e defendem que "[...] como o ciclo de acesso a novos produtos começa com os ricos e se estende aos pobres após um tempo mais ou menos longo (e que nem sempre se completa), há um aumento da desigualdade[...]” (p. 102).

Gibbons (2000), analisando a questão na sociedade americana, defende ponto de vista semelhante, destacando que a questão racial, além da educacional e da econômica é de grande importância. Assim como no Brasil se observa (SORJ et al., 2005) clara divisão digital entre brancos, pardos e negros, Gibbons (2000) mostra que existe igualmente uma divisão digital entre brancos, hispânicos e afroamericanos, estando estes no fim da fila da inclusão digital.

Determinar a exclusão ou inclusão digital simplesmente pela presença de um computador no domicílio e sua conexão à internet é uma simplificação, uma avaliação superficial da questão. Por um lado, os indivíduos que acessam a internet não o fazem somente de suas residências. $\mathrm{O}$ trabalho, as escolas, a casa de terceiros, lan house ou centros comunitários são pontos de uso importantes e frequentes (GIBBONS, 2000; SORJ et al., 2005; BRASIL, 2008). Além disso, em comunidades carentes da cidade do Rio de Janeiro, é famoso o fenômeno do "GatoNet", isto é, o acesso à internet (e televisão) a cabo de forma ilícita e que nem sempre é confessado.

Por outro lado, o fato de possuir computador na residência com acesso à internet não garante a inclusão digital do indivíduo. Fatores como nível educacional, renda, sexo e idade são determinantes nesse caso. A falta de habilidade é apontada por $50 \%$ dos indivíduos que têm computador, mas nunca acessaram a internet (BRASIL, 2010). Sorj \& Guedes (2005) observaram que o maior nível educacional e econômico, a faixa etária mais baixa e o sexo masculino estão relacionados ao maior uso do computador e da internet nas populaçôes de baixa renda. Nesse caso, a preponderância do sexo masculino se explica pelo tipo de atividade laboral. As mulheres dessas comunidades frequentemente trabalham no setor de limpeza (trabalho doméstico e limpeza), o que lhes dá menor oportunidade de acesso a computadores e internet no trabalho (SORJ et al., 2005). 
Outro ponto a ser destacado para a compreensão da inclusão/exclusão digital é a qualidade do acesso, tanto no que se refere ao tempo disponível para uso, quanto à qualidade do acesso em si. $\mathrm{O}$ custo da banda larga é alto. Ao mesmo tempo, por via discada, a qualidade da conexão é menor e o tempo precisa ser controlado, comprometendo a qualidade do uso. Existem ainda as restriçōes que a utilização no trabalho, na casa de terceiros e em centros comunitários acarretam. Gibbons (2000) destaca que, no caso da busca de informaçôes sobre saúde, quando a confidencialidade e o tempo disponível para pesquisa são muito importantes, o acesso fora da residência pode não ser o ideal.

\section{Considerações finais}

A internet é um recurso excepcional no que se refere à obtenção de informações de qualquer tipo, e pode ser um grande instrumento educacional. $\mathrm{O}$ conceito de tempo e espaço foi profundamente modificado por ela e por meio da grande rede mundial é possível ter acesso a diversas fontes de informação, como revistas científicas, universidades, hospitais, centros de pesquisa, entre muitas outras. É possível pesquisar tais fontes de forma rápida, fácil, a qualquer hora, de qualquer lugar.

Mas não para todos, ou talvez não ainda, e nem em qualquer lugar. Em primeiro lugar, são diferentes as possibilidades de cada grupo social de acessar a grande rede mundial. No Brasil, em torno de $50 \%$ da população nunca fez uso dessa moderna TIC, e, entre os que já o fizeram, muitos não têm acesso regular e fácil. Além disso, o simples fato de usar a internet não significa que o indivíduo tenha plena capacidade de compreensão das informaçóes encontradas, ou saiba como utilizá-las. Propostas eminentemente técnicas para a redução da divisão digital não dão conta do despreparo das populações em relação aos instrumentos necessários para usar a grande rede: "Apesar da importância atribuída à faceta libertária e democratizante da internet, são necessários pré-requisitos tecnoculturais para acessá-la" (CASTIEL et al., 2002 Pág. 311). É possível que a internet contribua para a reprodução de formas de exclusão sociais já existentes, que a divisão digital contribua para a manutenção das iniquidades na saúde.

$\mathrm{O}$ mesmo acontece com o tão propalado potencial de empowerment. Por um lado, a obtenção de informações técnicas e não técnicas (leigas) pode produzir 
no indivíduo ou na comunidade um maior controle sobre as questôes relativas

à saúde e ao ambiente, assim como uma redução da assimetria na relação com os profissionais da saúde. Por outro lado, esse mesmo fenômeno pode levar a um processo de responsabilização e culpabilização dos próprios indivíduos sobre sua saúde, sugerindo "que as pessoas façam, cada vez mais, uso de seus recursos próprios e/ou da comunidade antes de recorrer à ajuda de instituiçôes estatais” (CARVALHO, 2004, p.1091)

Considerando que a ciência (bio)médica hegemônica é a principal base teórico-cognitiva das informações na rede, o uso da internet pode vir a reforçar a intensa medicalização da sociedade, por meio de uma maior submissão ao modelo biomédico e a modelos sociais preestabelecidos.

Entretanto, apesar de todas as críticas acima, a internet pode vir a ser uma grande aliada na construção de projetos de promoção da saúde, em especial no que se refere ao desenvolvimento de habilidades pessoais. A instalação de laboratórios de informática em centros de saúde e em comunidades de baixa renda para utilização por grupos de usuários para educação em saúde, como um exemplo de proposta prática, pode representar uma alternativa válida e necessária. No desenvolvimento desses projetos, contudo, é fundamental considerar os potenciais riscos e compreender que as estratégias e programas na área da promoção da saúde precisam adaptar-se às necessidades e possibilidades de cada local e levar em conta as diferenças sociais, culturais e econômicas ${ }^{1}$.

\section{Referências}

BECHARA, M. Os espaços públicos de acesso à internet. In: BRASIL. Pesquisa sobre o Uso das Tecnologias da Informação e da Comunicação no Brasil: TIC Domicílios e TIC Empresas 2007. 2. ed. São Paulo: Comitê Gestor da Internet no Brasil, 2008.

BENIGERI, M.; PLUYE, P. Shortcomings of health information on the internet. Health Promotion International, v.18, n.4, 2003.

BERGER, M.; WAGNER, T.H.; BAKER, L.C. internet use and stigmatized illness. Soc Sci Med, v.61, n.8, p.1821-1827, 2005.

BRASIL. Ministério da Saúde. As cartas da Promoção da Saúde. Brasília: Ministério da Saúde, 2002. Disponível em: <www.saude.gov.br/bvs/conf_tratados.html>.

BRASIL. Pesquisa sobre o Uso das Tecnologias da Informação e da Comunicação no Brasil: TIC Domicílios e TIC Empresas 2007. 2.ed. São Paulo: Comitê Gestor da Internet no Brasil, 2008. 
BRASIL. Pesquisa sobre o Uso das Tecnologias da Informação e da Comunicação no Brasil: TIC Domicílios e TIC Empresas 2009. Brito]. São Paulo: Comitê Gestor da Internet no Brasil, 2010.

BROOM, A.; TOVEY, P. The role of the internet in cancer patients' engagement with complementary and alternative treatments. Health, v.12, n.2, p.139-155, 2008.

BUNDORF, M.K. et al. Who Searches the internet for Health Information? Health Services Research, v.41, n.3, Part I, June 2006.

BUSS, P.M. A saúde e seus determinantes sociais. PHYSIS: Rev. Saúde Coletiva, Rio de Janeiro, v.17, n.1, p.77-93, 2007.

. Promoção de saúde e qualidade de vida. Ciência \& Saúde Coletiva, v.5, n.1, p.163$177,2000$.

- Uma introdução ao conceito de promoção da saúde. In: CZERESNIA, D.; FREITAS, C.M. (Org.). Promoção da saúde: conceitos, reflexôes, tendências. Rio de Janeiro: Fiocruz, 2003. p.39-53.

CARVALHO, S.R. Os múltiplos sentidos da categoria “empowerment" no projeto de promoção à saúde. Cad. Saúde Pública, Rio de Janeiro, v.20, n.4, p.1088-1095, jul-ago, 2004. CASTELLS, M. The Rise of the Network Society. Cambridge, MA: Blackwell, 1996.

CASTIEL, L.D.; VASCONCELLOS-SILVA, P.R. internet e o autocuidado em saúde: como juntar os trapinhos? História, Ciências, Saúde, v.9, n.2, p.291-314, maio-ago. 2002.

CASTIEL, L.D.; VASCONCELLOS-SILVA, P.R. A interface internet/S@úde: perspectives e desafios. Interface - Comunicação, Saúde, Educação, v.7, n.13, p.47-64, 2003.

CONRAD, P. Medicalization and social control. Annual Review of Sociology, v.18, p.209$232,1992$.

The medicalization of society: on the transformation of human conditions into treatable disorders. Baltimore: The John Hopkins University Press, 2007.

COTTON, S.R.; GUPTA, S.S. Characteristics of online and offline health information seekers and factors that discriminate between them. Soc Sci Med, v.59, n.9, p.1795-1806, 2004. CUNHA, M.B. Das bibliotecas convencionais às digitais: diferenças e convergências. Perspectivas em Ciências da Informação, v.13, n.1, p.2-17, 2008.

CZERESNIA D. O conceito de saúde e a diferença entre prevenção e promoção. In: CZERESNIA, D.; FREITAS, C.M. (Org.). Promoção da saúde: conceitos, reflexões, tendências. Rio de Janeiro: Fiocruz, 2003. p. 39-53.

EYSENBACH, G., JADAD, A.R. Evidence-based Patient Choice and Consumer health informatics in the internet age. J Med internet Res, v.3, n.2, p.19, 2001. 
FOX, N.J.; WARD, K.; O'ROURKE, A. The "expert patient": empowerment or medical

dominance? The case of weight loss, pharmaceutical drugs and the internet. Soc Sci Med, v.60, n.6, p.1299-1309, 2005a.

FOX, N.J.; WARD, K.; O'ROURKE, A. Pro-anorexia, weight-loss drugs and the internet: an 'anti-recovery' explanatory model of anorexia. Sociology of Health and Illness, v.27, n.7, p.944-971, 2005b.

FUNDAÇÃO GETÚllo VARGAS. Mapa da Exclusão Digital. Rio de Janeiro: FGV/ IBRE, 2003. 143p.

GIBBONS, M.C. A Historical Overview of Health Disparities and the Potential of eHealth Solutions. J Med internet Res, v.7, n.5, p.50, 2005.

GIDDENS, A. Modernidade e Identidade. Rio de Janeiro: Jorge Zahar, 2002.

GILLETT, J. Media activism and internet use by people with HIV/AIDS. Sociology of Health and Illness, v.25, n.6, p.608-624, 2003.

GINMAN et al. Health communication and knowledge construction. Health Informatics Journal, v.9, n.4, p.301-313, 2003.

GRIERSON, T. et al. Using the internet to Build Community Capacity for Healthy Public Policy. Health Promot Pract, v.7, n.13, 2006.

HARDEY, M. Doctor in the house: the internet as a source of lay health knowledge and the challenge to expertise. Sociology of Health \& Illness, v.21, n.6, p.820-835, 1999.

HARDEY, M. "The story of my illness": personal accounts of illness on the internet. Health, v.6, n.1, p.31-46, 2002.

HART, K. Notes towards an anthropology of the internet. Horizontes Antropológicos, Porto Alegre, v.10, n.21, p.15-40, 2004.

INSTITUTO BRASILEIRO DE GEOGRAFIA E ESTATÍSTICA. Pnad 2004. http:// www.ibge.gov.br/. Acesso em: 11 set 2008.

KORP, P. Health on the internet: implications for health promotion. Health Education Research, v.21, n.1, p.78-86, 2006.

LÉVY, P. Tecnologias da Inteligência: o futuro do pensamento na era da informática. Rio de Janeiro: Ed 34, 1993, 208p (Coleção TRANS).

MARCONDES, W.D. A convergência de referências na promoção da saúde. Saúde e Sociedade, v.13, n.1, p.5-13, 2004.

NETTLETON, S. The Emergence of E-Scaped Medicine? Sociology, v.38, n.4, p.661-679, 2004.

NETTLETON, S.; BURROWS, R.; O’MALLEY, L. The mundane realities of the everyday lay use of the internet for health, and their consequences for media convergence. Sociology of Health \& Illness, v.27, n.7, p.972-992, 2005. 
PANDEY, S.K.; HART, J.J.; TIWARY, S. Women's health and the internet: understanding emerging trends and implications. Social Science \& Medicine, v.56, n.1, p.179-191, 2003.

QUEIRÓZ, M.S. O paradigma mecanicista da medicina ocidental moderna: uma perspectiva antropológica. Rev Saúde Pública, v.20, n.4, p.309-317, 1986.

ROSEN, G. A evolução da medicina social. In: NUNES E.D. (org). Medicina Social: aspectos históricos e teóricos. São Paulo: Global, 1983.

SEALE, C. New directions for critical internet health studies: representing cancer experience on the web. Sociology of Health and Illness, v.27, n.4, p.515-540, 2005.

SILVA, W.M. Navegar épreciso: avaliação de impactos do uso da internet na relação médicopaciente, 2006. Dissertação (Mestrado em Saúde Pública) - Faculdade de Saúde Pública, Universidade de São Paulo, 2006.

SORJ, B.; GUEDES, L.E. Exclusão digital: problemas conceituais, evidências empíricas e políticas públicas. Novos Estudos, n.72, 2005.

ZIEBLAND, S. The importance of being expert: the quest for cancer information on the internet. Soc Sci Med., v.59, n.9, p.1783-1793, 2004 a.

ZIEBLAND, S. et al. How the internet affects patients' experience of cancer: a qualitative study. BMJ, v.328, p.564- 569, 2004 b.

ZOLA, I.K. Medicine as an Institution of Social Control. Sociological Review, v.20, p.487504, 1972.

\section{Nota}

${ }^{1}$ H.B. da R. Garbin participou da concepção e redação do artigo; M.C.R. Guilam e A. Pereira Neto participaram da redação do artigo. 


\section{Abstract}

Internet and health promotion: a tool for the development of individual and social skills The development of individual and social skills, in the sense of acquiring greater control and produce empowerment, is considered a very important Health Promotion strategy. Access to information is one of the bases for such process. Much has been said and written about the immense potential of the internet, as studies point it as a special tool to obtain information and develop skills, both individual and communal. This article is based on national and international scientific literature on Health Promotion, Health Information on the internet and Digital Divide, with the aim of discussing the possibility of using the internet as a resource on Health Promotion projects, highlighting strength points and potential barriers, especially the digital divide. The authors believe that the internet may be a great ally in building projects for Health Promotion, however, in developing these projects is essential to consider the potential risks and understand that the strategies and programs in health promotion area need to adapt to the needs and possibilities of each site and take into account social, cultural and economic differences.

> Key words: health promotion; internet; individual and social skills. 